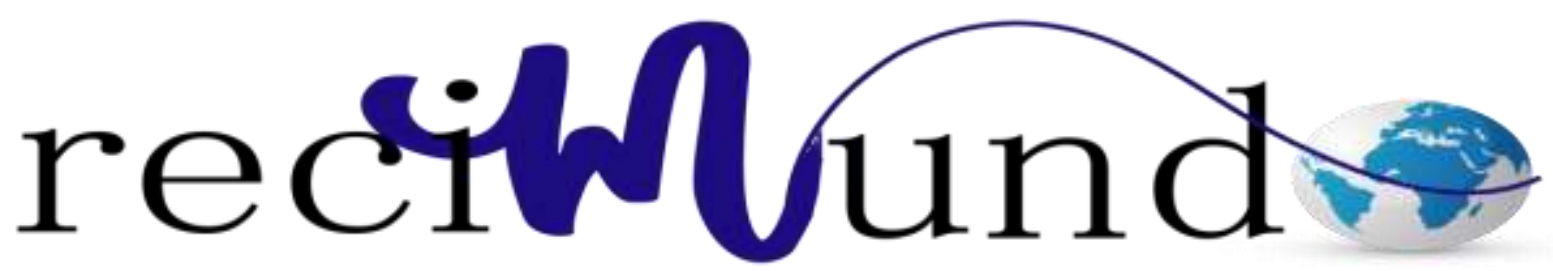

Revista Cientifica Mundo de la Investigación y el Conocimiento

Mariella Ginela Parrales Higuera ${ }^{a}$; Verónica Alexandra Merchán Jácome ${ }^{\text {b; }}$

Washington Enrique Pazmiño Gavilanez ${ }^{\text {c; }}$ Lorena Isabel Muñoz Oviedo ${ }^{\mathrm{d}}$

Modelo econométrico de la incidencia del Producto Interno Bruto con relación agregados monetarios, tasa de interés e inflación en el periodo 1990 -2018 de

Ecuador mediante la aplicación del software Gretl

Economic model of the incidence of the Gross Domestic Product with relation monetary aggregates, interest rate and inflation in the period 1990-2018 of

Ecuador through the application of Gretl software

Revista Científica Mundo de la Investigación y el Conocimiento. Vol. 3 núm.3. Esp., noviembre, ISSN: 2588-073X, 2019, pp. 876-905

DOI: $10.26820 /$ recimundo/3.(3.Esp).noviembre.2019.876-905

URL: http://recimundo.com/index.php/es/article/view/634

Código UNESCO: 5311.02 Gestión Financiera

Tipo de Investigación: Artículo de Revisión

(C) RECIMUNDO; Editorial Saberes del Conocimiento, 2019

Recibido: 15/09/2019 Aceptado: 23/10/2019_ Publicado: 30/11/2019

Correspondencia: maly2367@hotmail.com

a. Magister en Administración de Empresas; Ingeniera Comercial, Docente Investigador de la Facultad de Administración y Finanzas; Universidad Técnica de Babahoyo; Babahoyo, Ecuador; maly2367@hotmail.com - mparrales@utb.edu.ec

b. Magister en Administración de Empresas; Diploma Superior en Docencia Universitaria; Economista; Docente Investigador de la Facultad de Administración y Finanzas; Universidad Técnica de Babahoyo; Babahoyo, Ecuador; veronicamerchanjacome@yahoo.com vmerchan@utb.edu.ec

c. Magister Ejecutivo en Dirección de Empresas con Énfasis en Gerencia Estratégica; Ingeniero Comercial; Docente Investigador de la Facultad de Administración y Finanzas; Universidad Técnica de Babahoyo; Babahoyo, Ecuador; wpazmino@utb.edu.ec

d. Magister en Administración de Empresas; Diploma Superior en Docencia Universitaria; Ingeniera Comercial; Docente Investigador de la Facultad de Administración y Finanzas; Universidad Técnica de Babahoyo; Babahoyo, Ecuador; marigin@hotmail.es - $\underline{\text { lmunoz@utb.edu.ec }}$ 


\section{Modelo econométrico de la incidencia del Producto Interno Bruto con relación agregados monetarios, tasa de interés e inflación en el periodo 1990 - 2018 de Ecuador mediante la aplicación del software Gretl}

Vol. 3, núm. 3 Esp., (2019)

Mariella Ginela Parrales Higuera; Verónica Alexandra Merchán Jácome; Washington Enrique Pazmiño Gavilanez; Lorena Isabel Muñoz Oviedo

\section{ABSTRACT}

Since 1990 in Ecuador fluctuations have been registered in the Ecuadorian economy for several years. The economy of Ecuador has positive and negative factors that triggered the economic crisis of 1999, which became known as the banking holiday that led to the 2000 exit of the circulation of the Ecuadorian Sucre and the adoption of a new currency of the American dollar. Central Bank of Ecuador (ECB) dramatic record changes of decrease in the Gross Domestic Product (GDP), inflation, money supply, interest rate and total liquidity. With the economic indicators an economic model is proposed to determine the incidence of GDP with the aggregated values, interest rate and inflation from 1990 to 2018. It is necessary to emphasize that the Ecuadorian economy grew in the Econ government. Rafael Correa who was president of the Republic of Ecuador in his mandate carried out works in the country such as: education, health, roads and hydroelectric. With The data obtained from the Central Bank of Ecuador and once established the econometric model is run on the Software Gretl in the first instance applies the ARIMA model, contrasts Dickey Fuller and finally the Johansen cointegration test to show which of the independent variables explains how best to Gross Domestic Product.

Palabras Claves: Economic crisis, Banking holiday, inflation, total money supply, Gross Domestic Product, Interest rate. 


\section{Modelo econométrico de la incidencia del Producto Interno Bruto con relación agregados monetarios, tasa de interés e inflación en el periodo 1990 -2018 de Ecuador mediante la aplicación del software Gretl}

Vol. 3, núm. 3 Esp., (2019)

Mariella Ginela Parrales Higuera; Verónica Alexandra Merchán Jácome; Washington Enrique Pazmiño Gavilanez; Lorena Isabel Muñoz Oviedo

\section{RESUMEN}

Desde 1990 en Ecuador se ha registrado fluctuaciones en la economía ecuatoriana durante varios años. La economía de Ecuador tiene aspectos positivos y negativos destacándose la crisis economía del 1999 conocido como el feriado bancario que con llevo para el 2000 la salida de circulación del sucre ecuatoriano y la adopción de una nueva moneda el dólar americano. El Banco Central del Ecuador (BCE) registro drásticos cambios de decrecimiento en el Producto Interno Bruto (PIB), inflación, oferta monetaria, tasa de interés y liquidez total. Con los indicadores económicos se plantea un modelo econométrico para determinar la incidencia del PIB con los valores agregados, tasa de interés e inflación desde 1990 al 2018. En necesario destacar que la economía ecuatoriana creció en el gobierno del Econ. Rafael Correa quien fue presidente de la República de Ecuador en su mandato realizado obras en el país como: educación, salud, vialidad e hidroeléctricas. Con los datos obtenidos del Banco Central del Ecuador y una vez establecido el modelo econométrico se correrá en el Software Gretl en primera instancia se aplica el modelo ARIMA, contrastes de Dickey Fuller y por último la prueba de Cointegración de Johansen para evidenciar cuál de las variables independientes explica de mejor forma al Producto Interno Bruto.

KeyWords: Crisis económica, Feriado bancario, inflación, liquidez total oferta monetaria, Producto Interno Bruto, Tasa de interés. 


\section{Modelo econométrico de la incidencia del Producto Interno Bruto con relación agregados monetarios, tasa de interés e inflación en el periodo 1990 - 2018 de Ecuador mediante la aplicación del software Gretl}

Vol. 3, núm. 3 Esp., (2019)

Mariella Ginela Parrales Higuera; Verónica Alexandra Merchán Jácome; Washington Enrique Pazmiño Gavilanez; Lorena Isabel Muñoz Oviedo

\section{Introducción.}

El presente trabajo de investigación busca determinar los principales aspectos positivos y negativos de la política monetaria de Ecuador mediante un modelo econométrico teniendo como variable dependiente el PIB y variables independientes la Tasa De Interés Nominal, Inflación, Oferta Monetaria y la Liquidez Total.

El análisis de la política monetaria mediante las decisiones y acciones del Banco Central del Ecuador (BCE), a raíz de la crisis económica de 1999, Ecuador perdió su soberanía monetaria y se implantó un modelo de tipo de cambio fijo denominado como la dolarización. Ecuador perdió su soberanía monetaria el sucre y adoptar la moneda el dólar americano es decir el BCE dejo de emitir una moneda en la circulación monetaria en el Estado ecuatoriano. El conjunto de la evidencia empírica presentada en este trabajo indica la presencia de relaciones de diversa índole entre los agregados monetarios, la inflación y el PIB. Con objeto de identificar con mayor precisión la lógica económica inherente a estas relaciones se procedió a estimar un modelo econométrico.

Con la investigación se pretende evaluar las variables macroeconómicas mediante modelo econométrico con el fin de evaluar la estacionalidad de los datos a través del Test de Dickey Fuller posteriormente se corre el modelo a través del modelo Arima con predicciones posteriores a la base de datos es decir del año 2015 al año 2024. Como último procedimiento se evaluó la cointegración de las variables dentro del modelo a través del Método De Johansen presentando los resultados que posteriormente se analizará. 


\section{Modelo econométrico de la incidencia del Producto Interno Bruto con relación agregados monetarios, tasa de interés e inflación en el periodo 1990 -2018 de Ecuador mediante la aplicación del software Gretl}

Vol. 3, núm. 3 Esp., (2019)

Mariella Ginela Parrales Higuera; Verónica Alexandra Merchán Jácome; Washington Enrique Pazmiño Gavilanez; Lorena Isabel Muñoz Oviedo

\section{Materiales y métodos.}

\section{Modelo arima}

El modelo ARIMA se creó en 1970 por George Box y Gwilym Jenkins con la finalidad de describir los cambios en la serie temporal, aplicando un enfoque matemático. El modelo se basa en el ajuste de los valores observados, con el objetivo de reducir a cerca de cero la diferencia de los valores producidos en el modelo y los valores observados. Con el modelo Arima se puede describir el comportamiento de las series estacionarias y no estacionarias, otorgando versatilidad a una variedad de situaciones (Renato, 2013). Se suele expresar como ARIMA (p, d, q) donde los parámetros p, d y q son números enteros no negativos que indican el orden de las distintas componentes del modelo (Gujarati \& Porter, 2013).

\section{Cointegración}

Es una combinación lineal de variables no estacionarias, al realizar las diferencias a las variables de estudio se está realizando una cointegración. Desde el punto de vista económico se dicen que dos o más series están centígradas si las mismas se mueven conjuntamente a lo largo del tiempo y las diferencias entre ellas son estables (es decir estacionarias), aun cuando cada serie en particular contenga una tendencia estocástica y sea por lo tanto no estacionaria (Wooldridge, 2010). 


\section{Modelo econométrico de la incidencia del Producto Interno Bruto con relación agregados monetarios, tasa de interés e inflación en el periodo 1990 - 2018 de Ecuador mediante la aplicación del software Gretl}

Vol. 3, núm. 3 Esp., (2019)

Mariella Ginela Parrales Higuera; Verónica Alexandra Merchán Jácome; Washington Enrique Pazmiño Gavilanez; Lorena Isabel Muñoz Oviedo

Prueba de cointegración de Johansen

La mayor parte de las series temporales son no estacionarias y las técnicas convencionales de regresión basadas en datos no estacionarios tienden a producir resultados espurios, Sin embargo, las series no estacionarias pueden estar cointegradas si alguna combinación lineal de las series llega a ser estacionaria. Es decir, la serie puede deambular, pero en el largo plazo hay fuerzas económicas que tienden a empujarlas a un equilibrio (Alonso, 2011).

Prueba de dickey-fuller aumentada (ADF)

Es una prueba de raíz unitaria para una muestra de una serie de tiempo. Es una versión aumentada de la prueba Dickey-Fuller para un conjunto más amplio y más complejo de modelos de series de tiempo. La estadística Dickey-Fuller Aumentada (ADF), utilizada en la prueba, es un número negativo. Cuanto más negativo es, más fuerte es el rechazo de la hipótesis nula de que existe una raíz unitaria para un cierto nivel de confianza (Montero, 2013).

Series de tiempo estacionarias

Una serie es estacionaria cuando es estable a lo largo del tiempo, es decir, cuando la media y varianza son constantes en el tiempo. Esto se refleja gráficamente en que los valores de la serie tienden a oscilar alrededor de una media constante y la variabilidad con respecto a esa media también permanece constante en el tiempo (Santana \& Melo, 2016). 


\section{Modelo econométrico de la incidencia del Producto Interno Bruto con relación agregados monetarios, tasa de interés e inflación en el periodo 1990 -2018 de Ecuador mediante la aplicación del software Gretl}

Vol. 3, núm. 3 Esp., (2019)

Mariella Ginela Parrales Higuera; Verónica Alexandra Merchán Jácome; Washington Enrique Pazmiño Gavilanez; Lorena Isabel Muñoz Oviedo

Series de tiempo no estacionarias

Las series de tiempo no estacionarias tienen una tendencia y/o variabilidad cambian en el tiempo. Los cambios en la media determinan una tendencia a crecer o decrecer a largo plazo, por lo que la serie no oscila alrededor de un valor constante (Santana \& Melo, 2016).

\section{Resultados.}

Desarrollo del modelo econométrico

La política monetaria ecuatoriana puede incidir sobre el conjunto de la actividad económica a través de diversos canales de transmisión, tales como los agregados monetarios, las tasas de interés, el tipo de cambio o el crédito; es por ello que nuestro modelo se ve planteado sobre la incidencia que tiene los instrumentos de la política monetaria en el PIB ecuatoriano.

Teniendo como planteamiento el siguiente modelo econométrico para la investigación:

$$
\boldsymbol{Y}_{t}=\boldsymbol{R}_{t}+\boldsymbol{P}_{t}+\boldsymbol{M}_{\mathbf{1}}+\boldsymbol{M}_{2}
$$

Y: Producto Interno Bruto (PIB)

Rt: Tasa De Interés Nominal

Pt: Inflación

M1: Oferta Monetaria

M2: Liquidez Total

Análisis de las variables econométricas: 
Modelo econométrico de la incidencia del Producto Interno Bruto con relación agregados monetarios, tasa de interés e inflación en el periodo 1990 2018 de Ecuador mediante la aplicación del software Gretl

Vol. 3, núm. 3 Esp., (2019)

Mariella Ginela Parrales Higuera; Verónica Alexandra Merchán Jácome; Washington Enrique Pazmiño Gavilanez; Lorena Isabel Muñoz Oviedo

Variable independiente:

PIB: El Producto Interno Bruto está conformado por el mercado de bienes y servicios finales que produce un país en un año determinado. PIB es el indicador más amplio de la producción total de bienes y servicios de un Estado. Se compone por valores monetarios del consumo (C), la inversión bruta, compras de bienes y servicios del estado (G) y las exportaciones netas $(\mathrm{X})$ producidos en un país durante un determinado año formando el PIB.

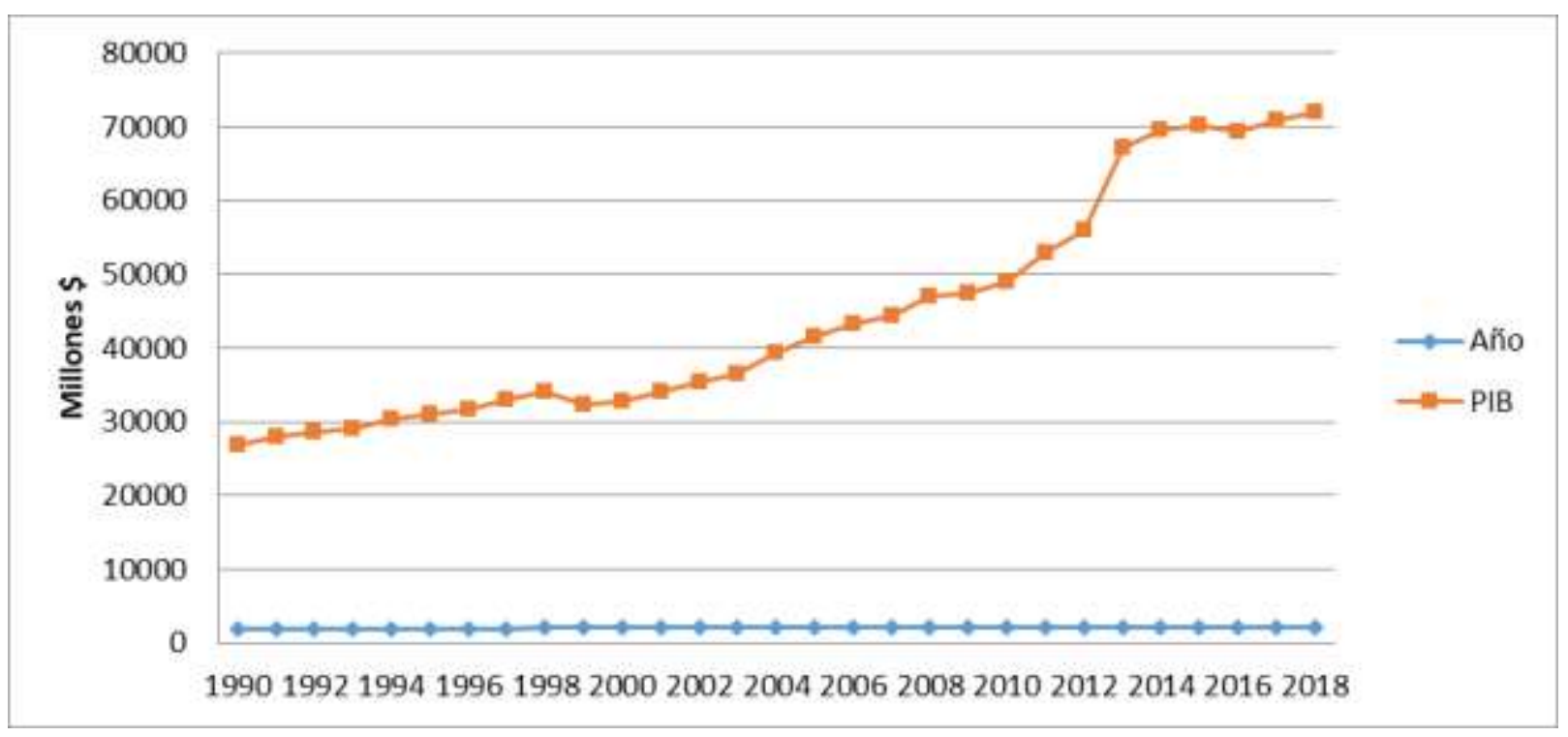

Fig. 1. PIB 1990 - 2018. Tomado del: Banco Central del Ecuador

En la Fig. 1 se evidencia que el PIB de Ecuador en la década de los noventa presenta un leve incremento hasta 1998 para 1999 la devaluación del sucre, y los altos niveles de endeudamiento y emisión monetaria, las variaciones del precio del petróleo y el cambio de moneda (dólar). El PIB creció a partir del año 2001 hasta el 2014, en el año 2015 hay una caída leve con 


\section{Modelo econométrico de la incidencia del Producto Interno Bruto con relación agregados monetarios, tasa de interés e inflación en el periodo 1990 -2018 de Ecuador mediante la aplicación del software Gretl}

Vol. 3, núm. 3 Esp., (2019)

Mariella Ginela Parrales Higuera; Verónica Alexandra Merchán Jácome; Washington Enrique Pazmiño Gavilanez; Lorena Isabel Muñoz Oviedo

respecto al 2014, sin embargo empieza un periodo de crecimiento sostenido desde el año 2016 hasta el 2018, en el gobierno del Econ. Rafael Correa hubo un crecimiento que va de 67.081 hasta 69.632 millones de dólares siendo estos los picos más altos en la historia del Ecuador en cuanto al PIB. Los resultados de las Cuentas Nacionales trimestrales publicados por el BCE, el PIB tuvo un crecimiento anual de $4.5 \%$ en el año 2013 con respecto a 2012, ubicando al país como una de las economías con mejores resultados en la región (Banco Central del Ecuador, 2010).

Variables dependientes:

La tasa de interés nominal

Una tasa de interés nominal tiene un crecimiento en el monto de dinero, sin ajustar la moneda por inflación. La tasa de interés nominal no necesariamente significa un incremento en el poder adquisitivo (UNAM, 2013).

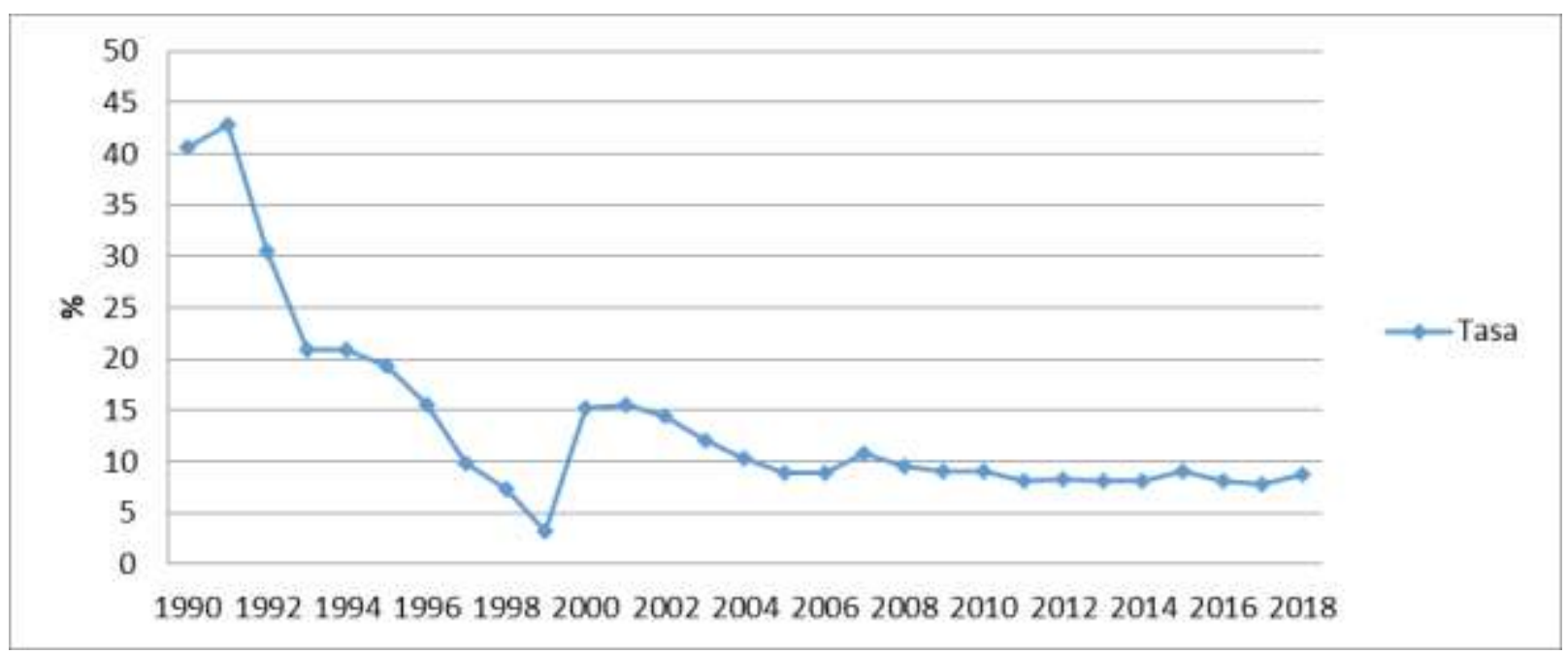

Fig. 2. Tasa de interés 1990 - 2018. Tomado del: Banco Central del Ecuador 


\section{Modelo econométrico de la incidencia del Producto Interno Bruto con relación agregados monetarios, tasa de interés e inflación en el periodo 1990 - 2018 de Ecuador mediante la aplicación del software Gretl}

Vol. 3, núm. 3 Esp., (2019)

Mariella Ginela Parrales Higuera; Verónica Alexandra Merchán Jácome; Washington Enrique Pazmiño Gavilanez; Lorena Isabel Muñoz Oviedo

En la Fig. 2 se evidencia que en la década de los noventa la tasa de interés fue alta teniendo en cuenta que a un se tenía moneda propia para 1999 la tasa de interés fue de 3.25\% siendo el año más bajo durante mucho tiempo. Para el 2001 subiría la tasa de interés al $15.53 \%$ y en los posteriores años decrecería paulatinamente siendo para el 2014 un valor de 8.13\%, en el año 2015 hay un leve aumento del 9,12\%, decreciendo en los años 2016 y 2017, volviendo a aumentar para el año 2018 al 8,69\%.

Oferta monetaria M1

Se define como la cantidad de dinero a disposición inmediata de los agentes para realizar transacciones; contablemente el dinero en sentido estricto, es la suma de las especies monetarias en circulación, la moneda fraccionaria y los depósitos en cuenta corriente (Banco Central del Ecuador, 2010).

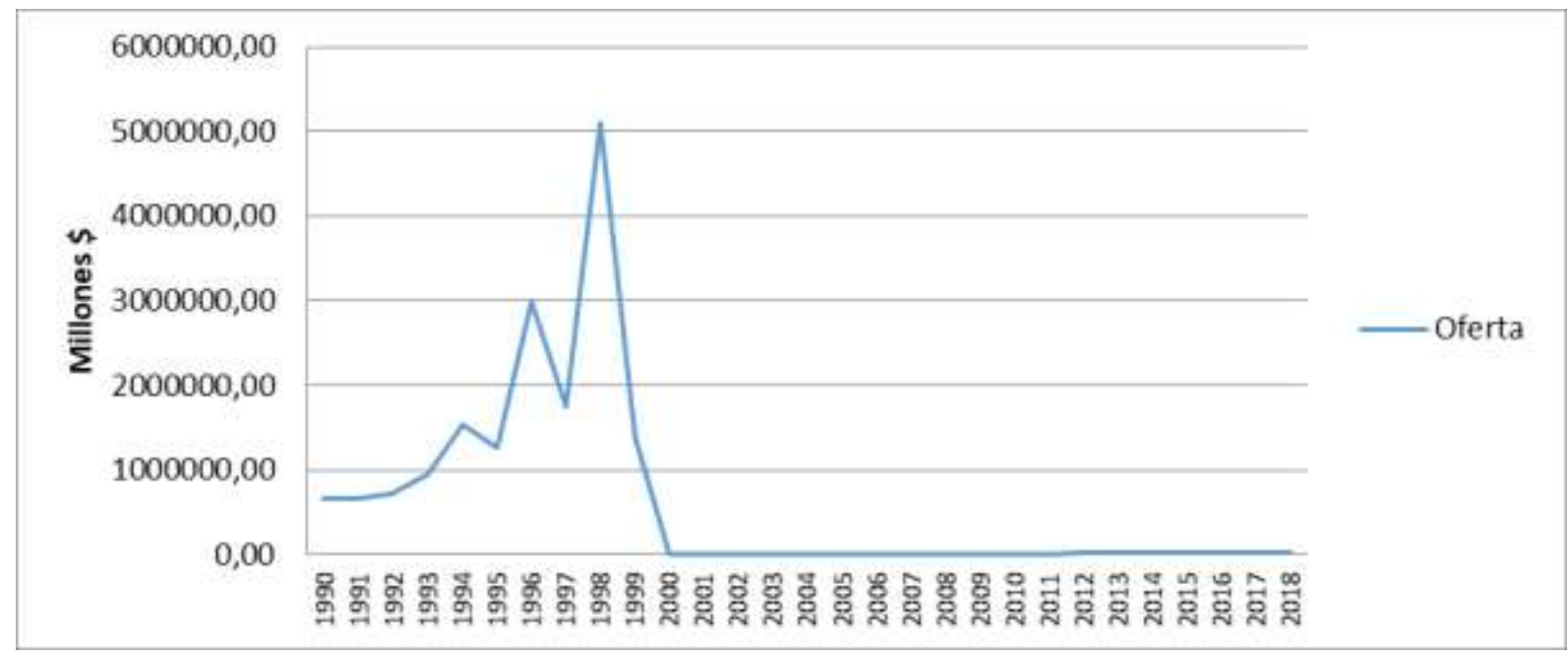

Fig. 3. Oferta Monetaria M1 1990 - 2018. Tomado del: Banco Central del Ecuador 


\section{Modelo econométrico de la incidencia del Producto Interno Bruto con relación agregados monetarios, tasa de interés e inflación en el periodo 1990 -2018 de Ecuador mediante la aplicación del software Gretl}

Vol. 3, núm. 3 Esp., (2019)

Mariella Ginela Parrales Higuera; Verónica Alexandra Merchán Jácome; Washington Enrique Pazmiño Gavilanez; Lorena Isabel Muñoz Oviedo

La oferta monetaria en la Fig. 3 se evidencia dos picos altos donde el BCE inyectó dinero en 1996 pero el año más relevante de la década de los noventa fue en 1998 aumentando notablemente la oferta monetaria. Cuando Ecuador perdió su moneda decreció la oferta monetaria, esto se debe porque adopto una moneda extranjera como es el dólar americano.

En la crisis de Ecuador el circulante fue de 544 millones para diciembre de 1999, mes anterior a la adopción oficial de la dolarización. Durante el proceso de canje se colocó en circulación aproximadamente USD 540 millones, lo que significó que al finalizar el año 2000 la economía ecuatoriana contó con una liquidez superior a USD 2.100 millones (Herrera \& Pérez Martínez, 2015).

Liquidez total M2

Está conformado por la suma de la oferta monetaria M1 y el cuasi dinero, es decir todos los depósitos y captaciones, que los sectores tenedores de dinero mantienen en el sistema financiero nacional. También se le conoce como dinero en sentido amplio (Banco Central del Ecuador, 2010). 


\section{Modelo econométrico de la incidencia del Producto Interno Bruto con relación agregados monetarios, tasa de interés e inflación en el periodo 1990 - 2018 de Ecuador mediante la aplicación del software Gretl}

Vol. 3, núm. 3 Esp., (2019)

Mariella Ginela Parrales Higuera; Verónica Alexandra Merchán Jácome; Washington Enrique Pazmiño Gavilanez; Lorena Isabel Muñoz Oviedo

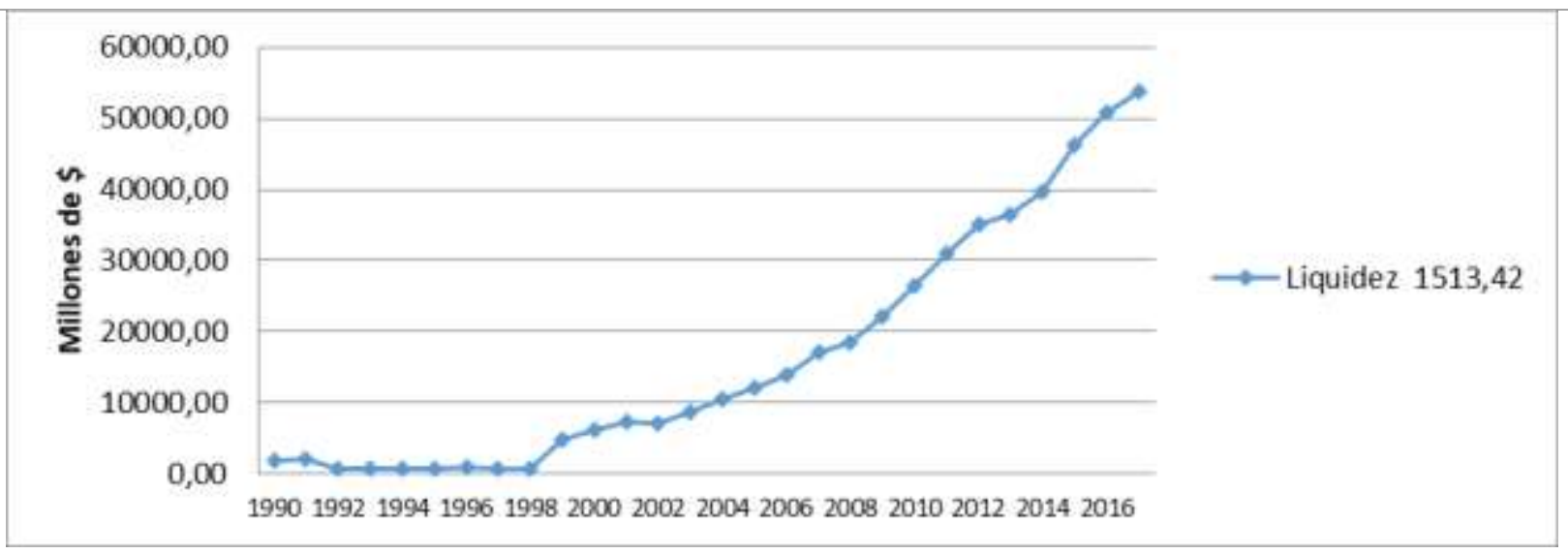

Fig. 4. Liquidez Total M2 1990 - 2018. Tomado del: Banco Central del Ecuador

En la Fig. 4 se evidencia entre 1992 a 1998 la liquidez total M2 decreció con referencia a 1991. Para 1998 la liquidez total ha ido creciendo anualmente es necesario destacar durante la crisis financiera ecuatoriana muchos ciudadanos ecuatorianos tuvieron que emigrar al extranjero y gracias a la ayuda de ellos también por medio de las remesas que enviaban a sus familiares al país han hecho que la liquidez del país crezca.

Inflación

Es el crecimiento continuo y generalizado de los precios de los bienes y servicios y factores productivos de una economía a lo largo del tiempo. La evolución de la inflación se mide por la variación del Índice de Precios al Consumidor (IPC). Para comprender el fenómeno de la inflación, se debe distinguir los aumentos generalizados de precios, que se producen y de aquellos aumentos de precios que son persistentes en el tiempo (García Camilo Pérez N, 2017). 


\section{Modelo econométrico de la incidencia del Producto Interno Bruto con relación agregados monetarios, tasa de interés e inflación en el periodo 1990 -2018 de Ecuador mediante la aplicación del software Gretl}

Vol. 3, núm. 3 Esp., (2019)

Mariella Ginela Parrales Higuera; Verónica Alexandra Merchán Jácome; Washington Enrique Pazmiño Gavilanez; Lorena Isabel Muñoz Oviedo

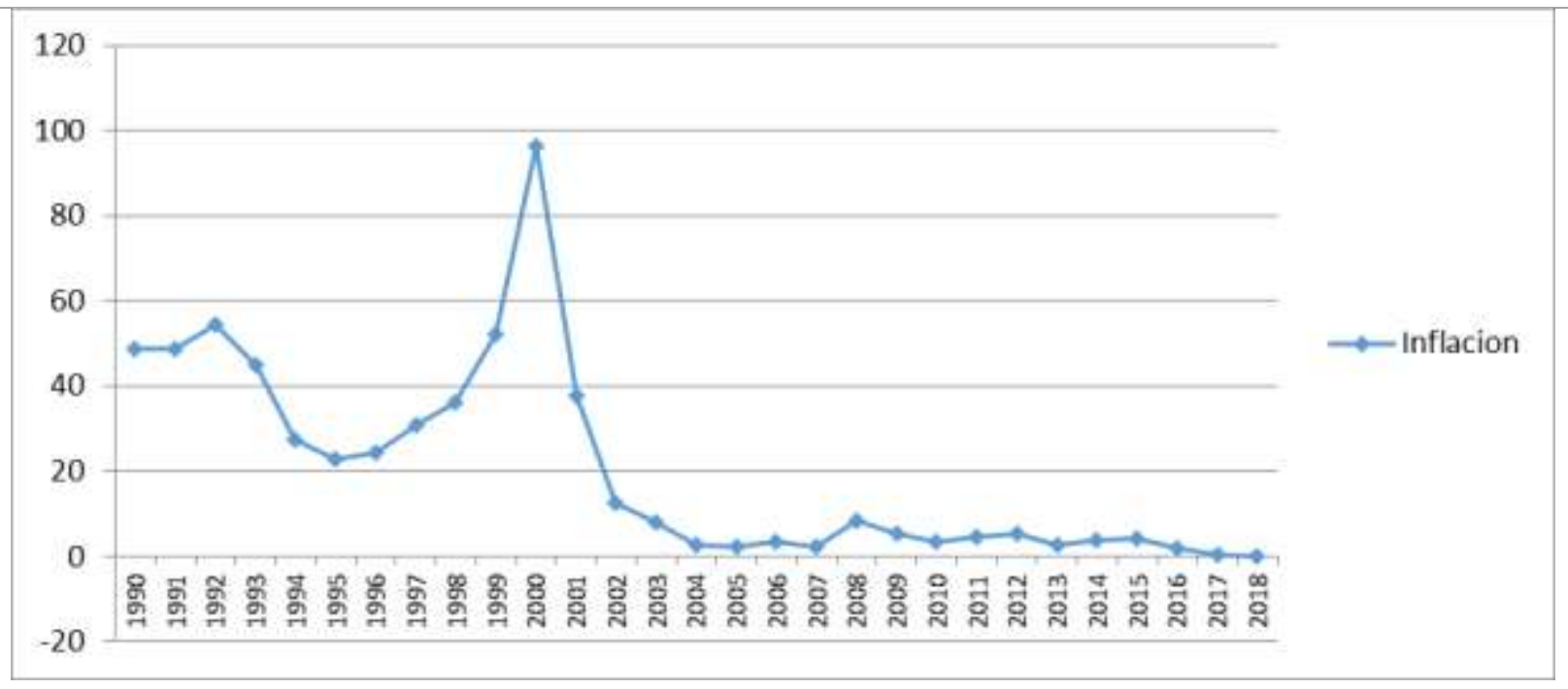

Fig. 5. Inflación 1990 -2018. Tomado del: Banco Mundial

En la Fig. 5 el pico más alto de la inflación fue de $96.1 \%$ en el 2000 como se mencionó anterior mente esto fue a consecuencia de la crisis económica que vivió el país, el cambio de moneda del sucre ecuatoriano al dólar americano, el desempleo, la depreciación de la moneda fueron factores para la inflación. En los años posteriores la inflación decreció a excepción del 2008 que la inflación creció en 8.83\%, del año 2009 al 2012 hubo cierta estabilidad inflacionaria, en el año 2013 hubo un decrecimiento al situarse en un 2,72\% con respecto al 2012 que hubo una inflación del 5,10\%, luego hubo leves incrementos en los años 2014 y 2015 y vuelve a observarse un decrecimiento más sostenido en los años 2016, 2017y 2018 situándose ese año prácticamente en inflación 0 con un $-0,22 \%$. 


\section{Modelo econométrico de la incidencia del Producto Interno Bruto con relación agregados monetarios, tasa de interés e inflación en el periodo 1990 - 2018 de Ecuador mediante la aplicación del software Gretl}

Vol. 3, núm. 3 Esp., (2019)

Mariella Ginela Parrales Higuera; Verónica Alexandra Merchán Jácome; Washington Enrique Pazmiño Gavilanez; Lorena Isabel Muñoz Oviedo

Otro factor principal con referencia a la inflación del 2000 fue el ajuste de la dolarización causando un impacto enorme en los precios de la canasta básica, servicios básicos, transporte y telecomunicaciones (Herrera \& Pérez Martínez, 2015).

Los datos de dicho modelo se lo presentan en una tabla dinámica con datos obtenidos del Banco Central de Ecuador, los mismos que serán la herramienta principal para la ejecución y solución del modelo.

\begin{tabular}{|c|c|c|c|c|c|c|}
\hline Moneda & Año & PIB & $\begin{array}{l}\text { Tasa de } \\
\text { interés } \\
\text { nominal }\end{array}$ & $\begin{array}{c}\text { Oferta } \\
\text { monetaria } \\
\text { M1 }\end{array}$ & $\begin{array}{l}\text { Liquidez } \\
\text { total M2 }\end{array}$ & Inflación \\
\hline \multirow{10}{*}{$\begin{array}{c}\text { Sucres } \\
\text { convertido } \\
\text { a dólares }\end{array}$} & 1990 & $26.804,00$ & 40,56 & $659.854,00$ & 1513.42 & 49,5 \\
\hline & 1991 & $27.955,00$ & 42,86 & $665.691,00$ & 1811,29 & 49 \\
\hline & 1992 & $28.546,00$ & 30,59 & $726.464,00$ & 2003,3 & 60,2 \\
\hline & 1993 & $29.109,00$ & 20,95 & $956.281,00$ & 790,89 & 31 \\
\hline & 1994 & $30.348,00$ & 20,89 & $1.543 .232,00$ & 814,97 & 25,4 \\
\hline & 1995 & $31.032,00$ & 19,3 & $1.267 .874,00$ & 824,28 & 22,8 \\
\hline & 1996 & $31.569,00$ & 15,52 & $2.982 .449,00$ & 844,33 & 25,6 \\
\hline & 1997 & $32.936,00$ & 9,85 & $1.748 .399,00$ & 932,51 & 30,7 \\
\hline & 1998 & $34.012,00$ & 7,36 & $5.106 .461,00$ & 839,15 & 43,3 \\
\hline & 1999 & $32.400,00$ & 3,25 & $1.401 .929,00$ & 667,18 & 60,7 \\
\hline \multirow[t]{12}{*}{ Dólares } & 2000 & $32.753,00$ & 15,17 & $2.092,19$ & 4874,7 & 96,1 \\
\hline & 2001 & $34.069,00$ & 15,53 & $2.964,66$ & 6157,14 & 37,7 \\
\hline & 2002 & $35.464,00$ & 14,36 & $3.703,01$ & 7452,66 & 12,5 \\
\hline & 2003 & $36.430,00$ & 12,03 & $3.936,11$ & 7176,62 & 7,9 \\
\hline & 2004 & $39.421,00$ & 10,39 & $4.586,43$ & 8678,55 & 3 \\
\hline & 2005 & $41.507,00$ & 8,84 & $5.410,96$ & 10451,4 & 2 \\
\hline & 2006 & $43.335,00$ & 8,85 & $6.259,79$ & 12083,54 & 2,87 \\
\hline & 2007 & $44.284,00$ & 10,82 & $7.009,21$ & 14013,27 & 3,32 \\
\hline & 2008 & $47.099,00$ & 9,57 & $8.880,31$ & 17177,26 & 8,83 \\
\hline & 2009 & $47.366,00$ & 9,1 & $9.209,67$ & 18588,46 & 4,31 \\
\hline & 2010 & $49.036,00$ & 9 & $10.776,15$ & 22189,37 & 3,33 \\
\hline & 2011 & $52.894,00$ & 8,15 & $12.088,08$ & 26550,19 & 5,41 \\
\hline
\end{tabular}


Modelo econométrico de la incidencia del Producto Interno Bruto con relación agregados monetarios, tasa de interés e inflación en el periodo 1990 -2018 de Ecuador mediante la aplicación del software Gretl

Vol. 3, núm. 3 Esp., (2019)

Mariella Ginela Parrales Higuera; Verónica Alexandra Merchán Jácome; Washington Enrique Pazmiño Gavilanez; Lorena Isabel Muñoz Oviedo

\begin{tabular}{ccccccc}
\hline 2012 & $55.878,00$ & 8,23 & $14.003,50$ & $30.906,00$ & 4,16 \\
\hline 2013 & $67.081,00$ & 8,17 & $15.548,90$ & 35051 & 2,7 \\
\hline 2014 & $69.632,00$ & 8,13 & $16.617,51$ & $36.465,03$ & 3,67 \\
\hline 2015 & $70.174,68$ & 9,12 & $19.041,70$ & $39.650,6$ & 3,97 \\
\hline 2016 & $69.314,07$ & 8,1 & $22.634,80$ & $46.188,3$ & 1,73 \\
\hline 2017 & $70.955,69$ & 7,83 & $24.530,50$ & $50.790,78$ & 0,42 \\
\hline 2018 & $71.932,84$ & 8,69 & $25.259,90$ & $53.664,74$ & $-0,22$ \\
\hline
\end{tabular}

Tabla I. Datos para correr el modelo econométrico en Gretl

Fuente: Banco Central del Ecuador

Elaborador por: Grupo Investigador

Aplicación de datos en el software gretl

Antes de proceder con el modelo econométrico Arima, se correrá por MCO el modelo para saber si es significativo el modelo planteado. 


\section{Modelo econométrico de la incidencia del Producto Interno Bruto con relación agregados monetarios, tasa de interés e inflación en el periodo 1990 - 2018 de Ecuador mediante la aplicación del software Gretl}

Vol. 3, núm. 3 Esp., (2019)

Mariella Ginela Parrales Higuera; Verónica Alexandra Merchán Jácome; Washington Enrique Pazmiño Gavilanez; Lorena Isabel Muñoz Oviedo

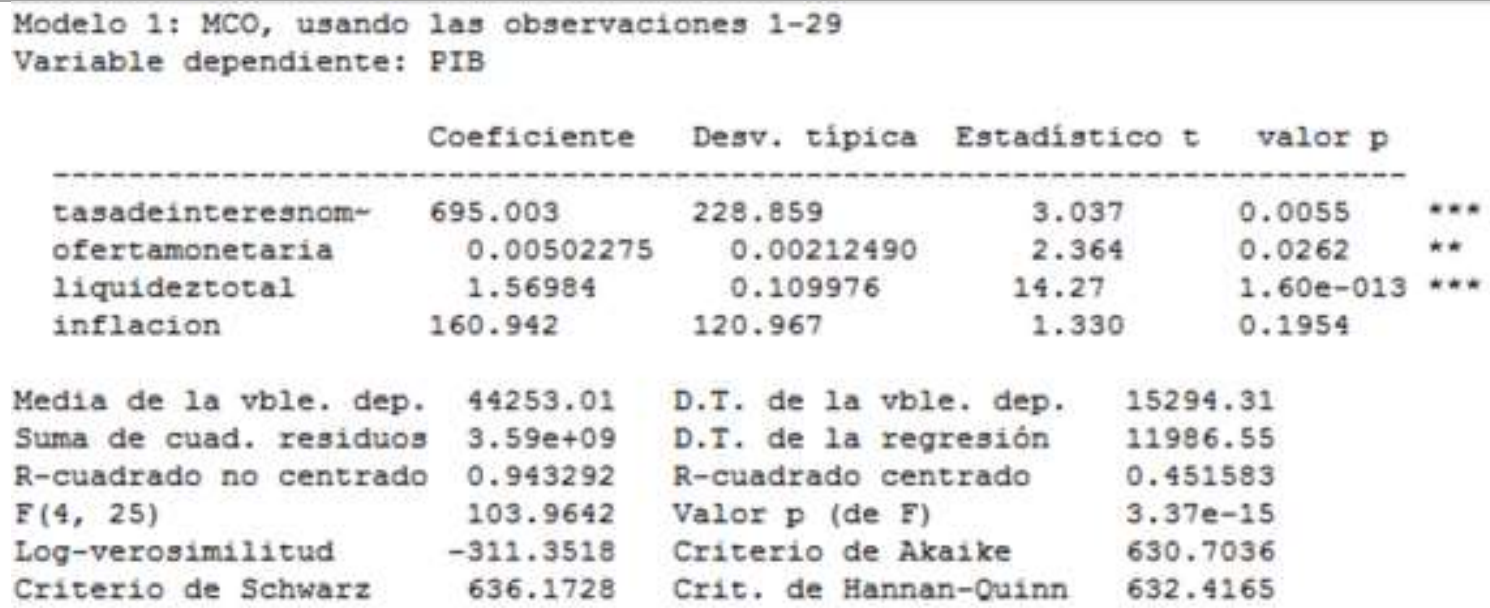

Fig. 6. Mínimos Cuadrados Ordinarios del modelo

En la Fig. 6 el modelo econométrico es muy significativo en todas sus variables independientes sobre todo en la variable de Liquidez Total, lo que significa que esta variable es la que explica mejor al modelo.

Producto interno bruto - PIB

Datos estacionarios

Para poder evaluar si los datos son o no estacionarios se procede a realizar el test de Dickey Fuller que se encuentra dentro del contraste de raíz unitaria. 


\section{Modelo econométrico de la incidencia del Producto Interno Bruto con relación agregados monetarios, tasa de interés e inflación en el periodo 1990 -2018 de Ecuador mediante la aplicación del software Gretl}

Vol. 3, núm. 3 Esp., (2019)

Mariella Ginela Parrales Higuera; Verónica Alexandra Merchán Jácome; Washington Enrique Pazmiño Gavilanez; Lorena Isabel Muñoz Oviedo

Test de dickey fuller

Con el Test de Dickey-Fuller trabajamos con la variable PIB. Para la ejecución de este apartado seleccionamos de retardos con la tercera parte del total de los datos es decir el total de datos es 29 entonces el número de retardos será 9,6 redondeando a 10 .

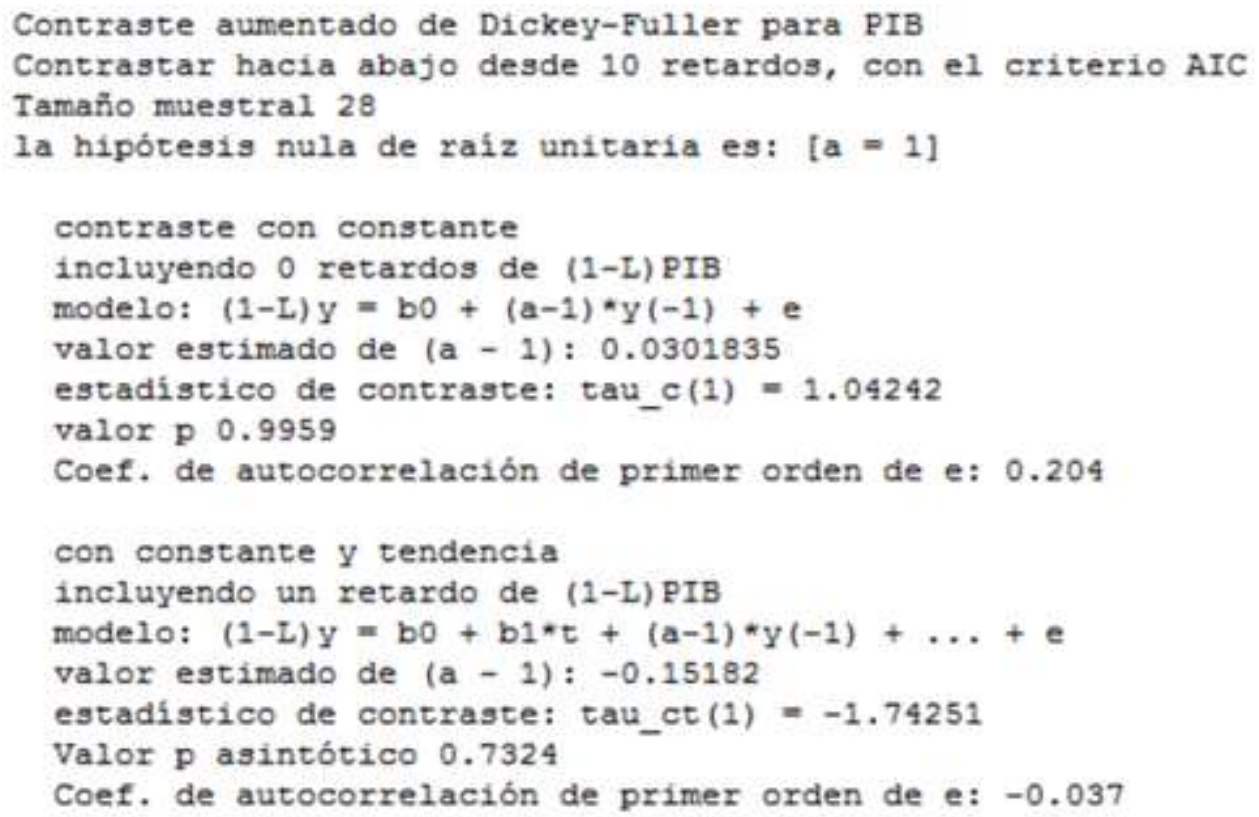

Fig. 7. Test de Dickey Fuller

Al realizar el Test de Dickey Fuller se tiene que la probabilidad de que el estimador Rho es estadísticamente igual a 0 , con un valor de probabilidad que se acerca a uno por lo que se acepta la hipótesis nula.

Ho: Los datos son estacionarios Test de Dickey-Fuller 


\section{Modelo econométrico de la incidencia del Producto Interno Bruto con relación agregados monetarios, tasa de interés e inflación en el periodo 1990 - 2018 de Ecuador mediante la aplicación del software Gretl}

Vol. 3, núm. 3 Esp., (2019) Mariella Ginela Parrales Higuera; Verónica Alexandra Merchán Jácome; Washington Enrique Pazmiño Gavilanez; Lorena Isabel Muñoz Oviedo

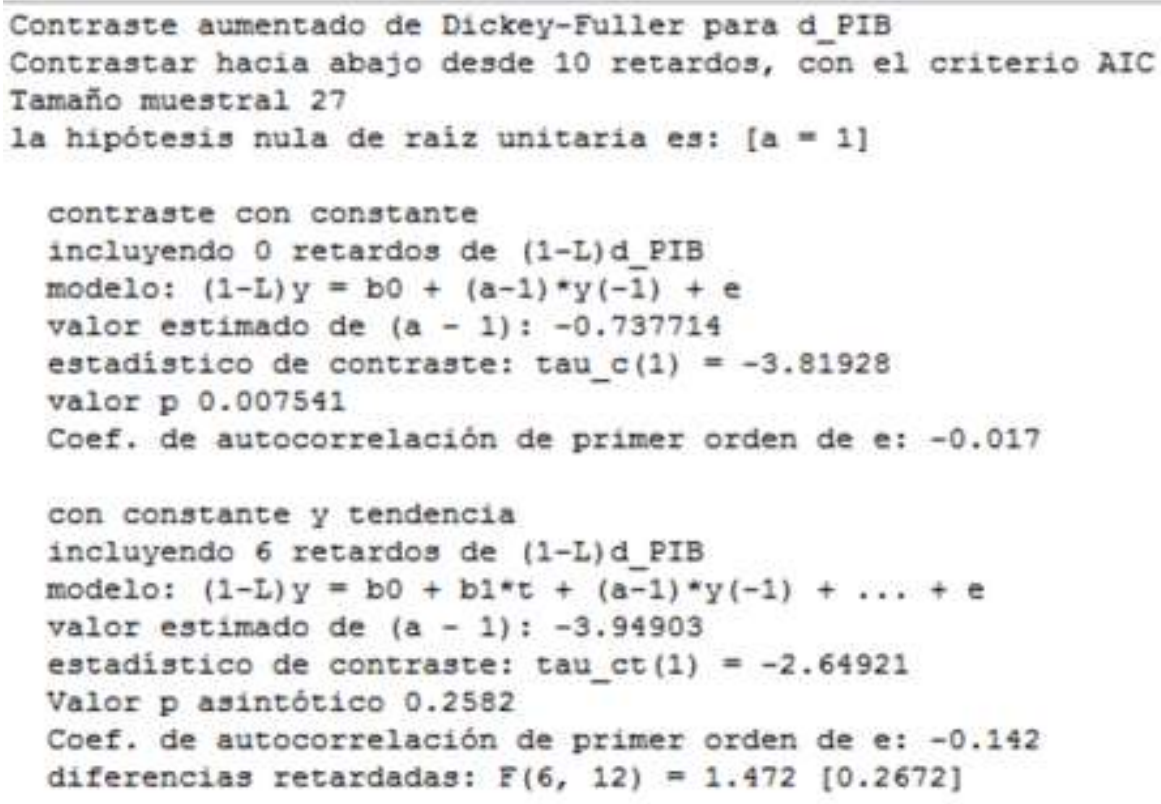

Fig. 8. Test de Dickey Fuller

Fuente: Gretl

Se puede observar que el estimador Rho es estadísticamente igual a 1, con un valor de probabilidad cercano a uno por lo que se rechaza la hipótesis nula. Por tanto, ya se obtuvo datos estacionarios. 


\section{Modelo econométrico de la incidencia del Producto Interno Bruto con relación agregados monetarios, tasa de interés e inflación en el periodo 1990 -2018 de Ecuador mediante la aplicación del software Gretl}

Vol. 3, núm. 3 Esp., (2019)

Mariella Ginela Parrales Higuera; Verónica Alexandra Merchán Jácome; Washington Enrique Pazmiño Gavilanez; Lorena Isabel Muñoz Oviedo

\section{Modelo arima}

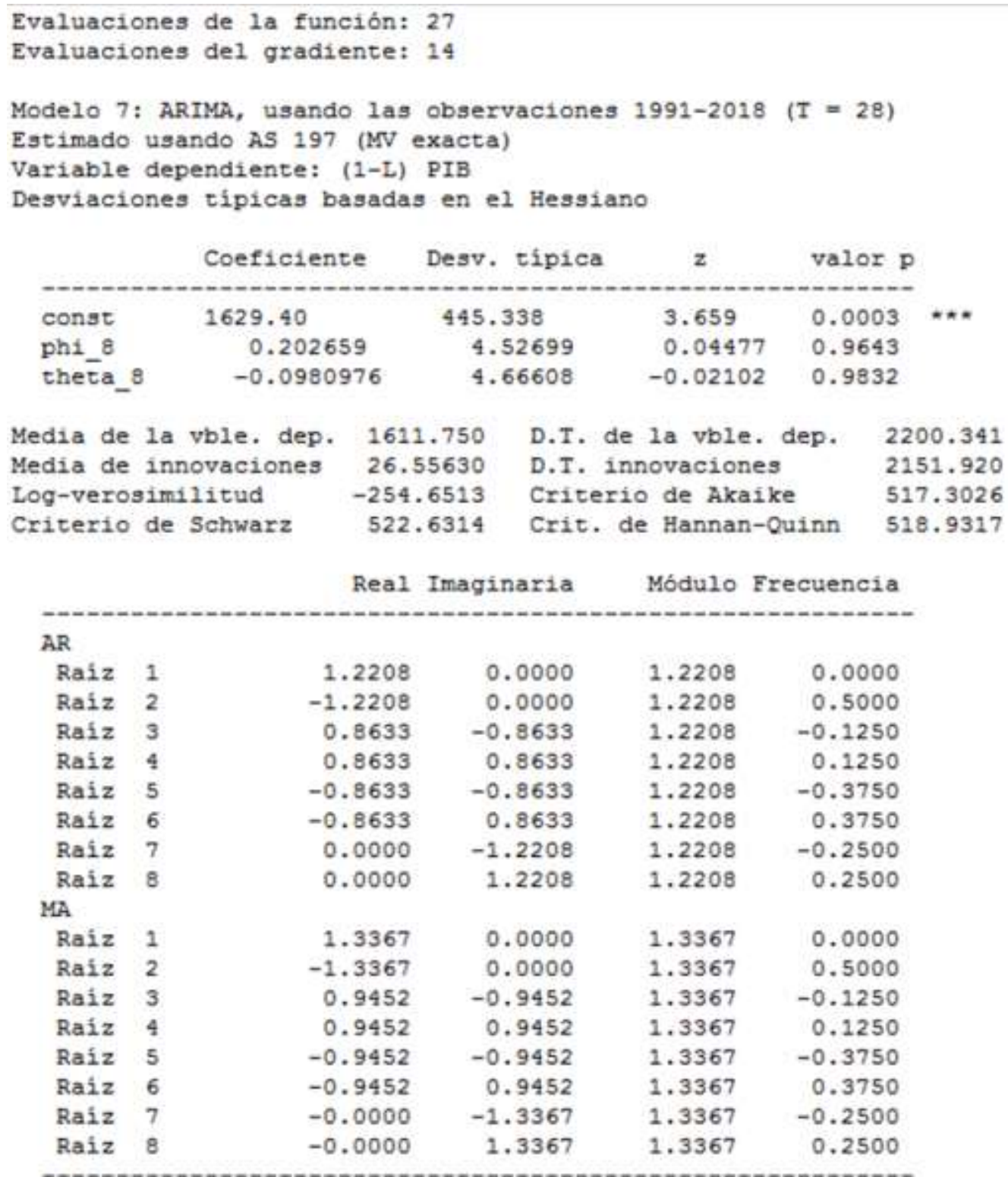

Fig. 9. Modelo ARIMA 
Modelo econométrico de la incidencia del Producto Interno Bruto con relación agregados monetarios, tasa de interés e inflación en el periodo 1990 2018 de Ecuador mediante la aplicación del software Gretl

Vol. 3, núm. 3 Esp., (2019)

Mariella Ginela Parrales Higuera; Verónica Alexandra Merchán Jácome; Washington Enrique Pazmiño Gavilanez; Lorena Isabel Muñoz Oviedo

Predicciones

El modelo Arima nos ayuda para analizar las predicciones futuras. Así como se muestra a continuación con la predicción de la variable PIB desde el año 2015 al 2021.

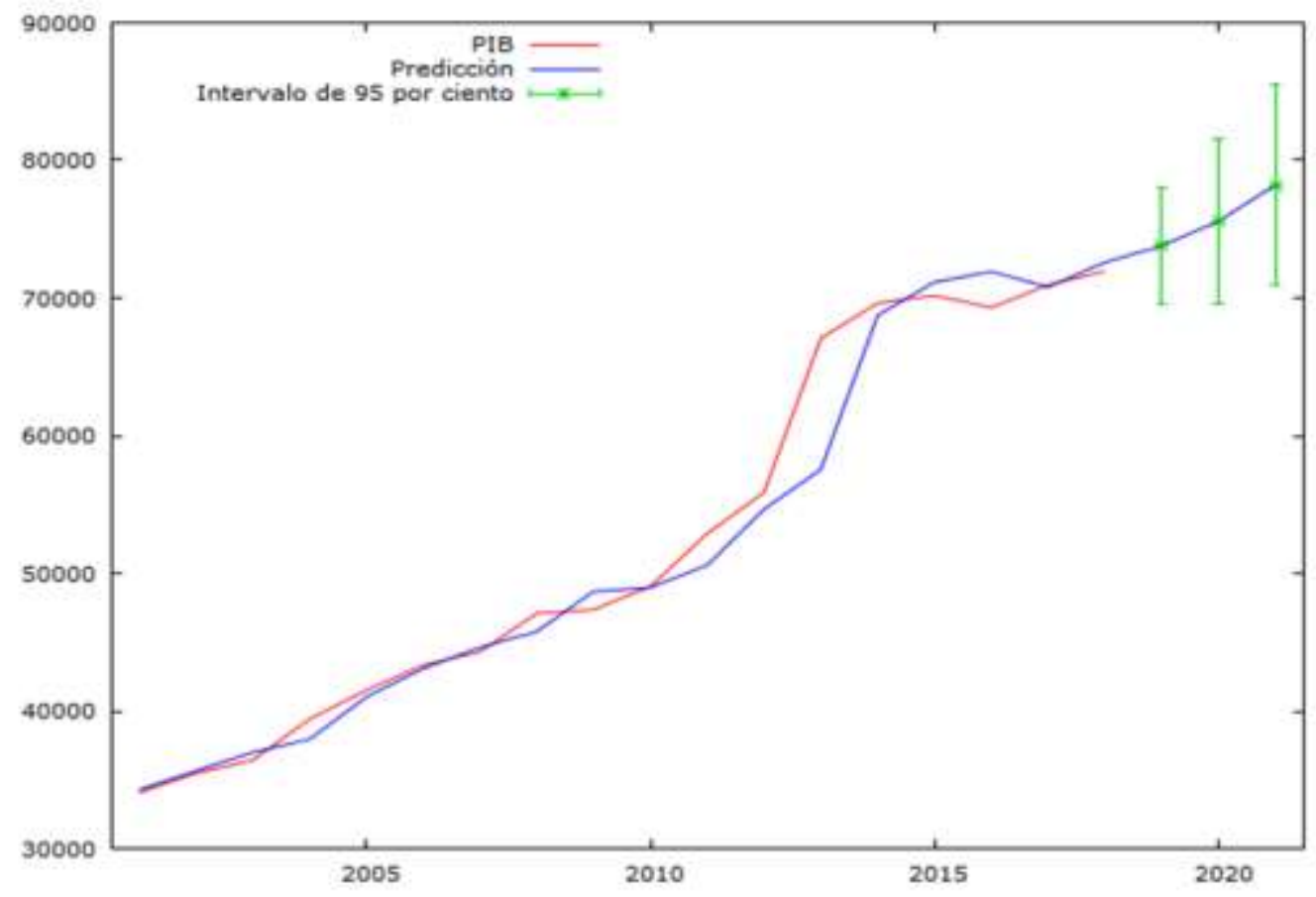

Fig. 10. Predicciones de la variable PIB

Como se puede observar las predicciones de la variable se analiza que la gráfica tiene una tendencia creciente a lo largo del tiempo. 


\section{Modelo econométrico de la incidencia del Producto Interno Bruto con relación agregados monetarios, tasa de interés e inflación en el periodo 1990 -2018 de Ecuador mediante la aplicación del software Gretl}

Vol. 3, núm. 3 Esp., (2019)

Mariella Ginela Parrales Higuera; Verónica Alexandra Merchán Jácome; Washington Enrique Pazmiño Gavilanez; Lorena Isabel Muñoz Oviedo

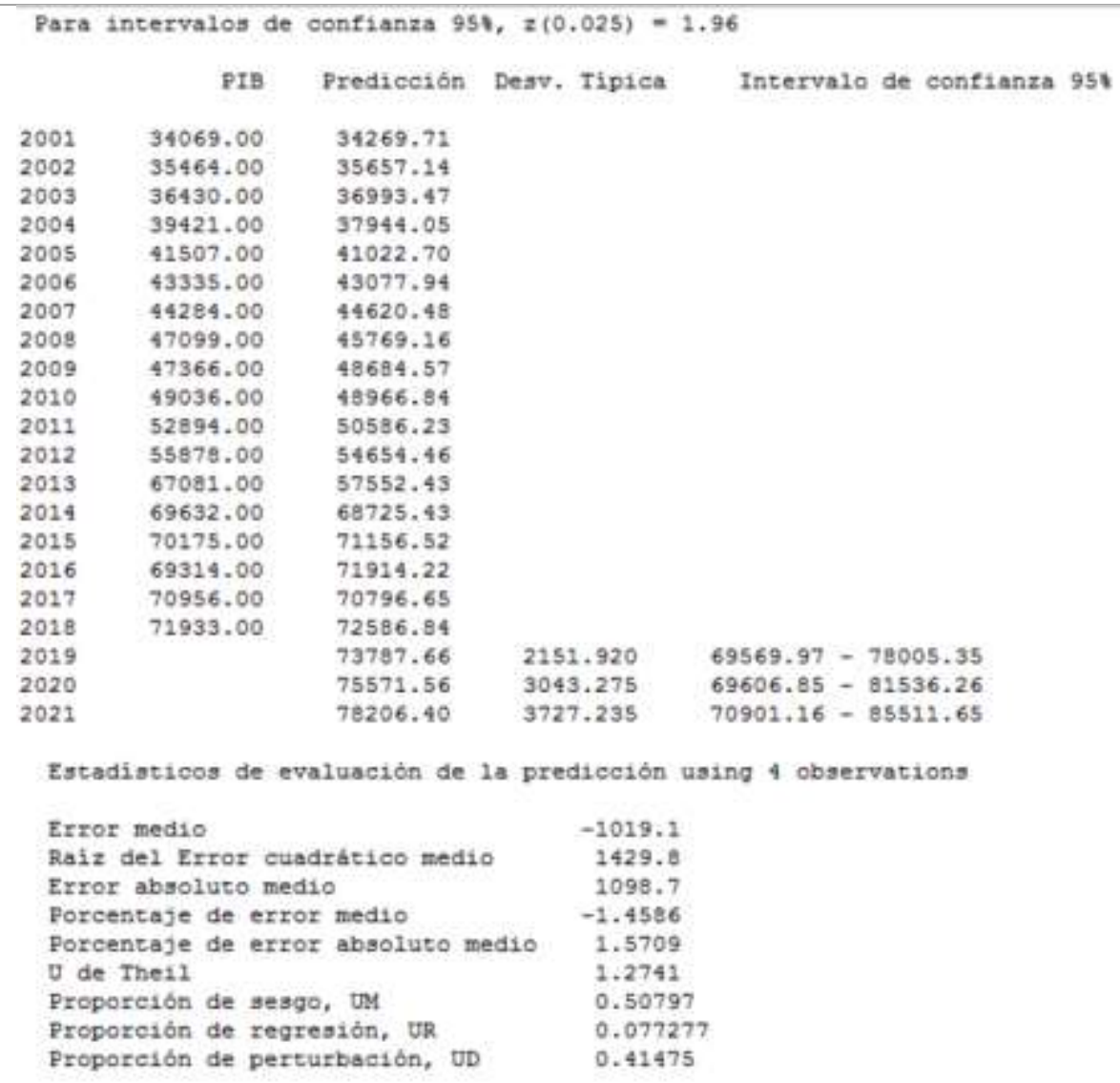

Fig. 11. Predicciones de la variable PIB

Se presenta los datos exactos de futuras predicciones de la variable PIB como se ha dicho anteriormente tienen una tendencia creciente.

\section{Cointegración}

Para analizar si existe cointegración en nuestro modelo utilizaremos el método de $\mathrm{S}$.

Johansen con el fin de determinar el número vectores de cointegración. 
Modelo econométrico de la incidencia del Producto Interno Bruto con relación agregados monetarios, tasa de interés e inflación en el periodo 1990 2018 de Ecuador mediante la aplicación del software Gretl

Vol. 3, núm. 3 Esp., (2019)

Mariella Ginela Parrales Higuera; Verónica Alexandra Merchán Jácome; Washington Enrique Pazmiño Gavilanez; Lorena Isabel Muñoz Oviedo

Método de johansen

En primera instancia para verificar si existe cointegración entre la variable dependiente y las variables independientes; procedemos a comprobar a través del gráfico de series temporales en el siguiente orden para el análisis:

PIB y Tasa de Interés Nominal

PIB y Oferta Monetaria

PIB y Liquidez Total

PIB y Tasa de Inflación

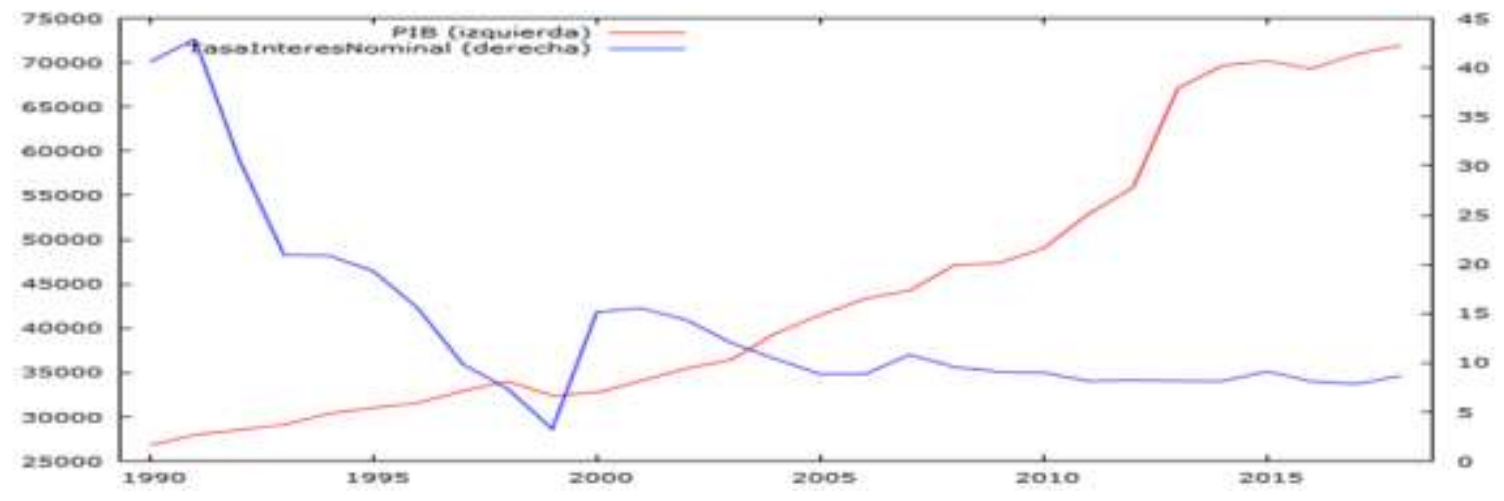

Fig. 12. PIB y Tasa de Interés Nominal 
Modelo econométrico de la incidencia del Producto Interno Bruto con relación agregados monetarios, tasa de interés e inflación en el periodo 1990 -2018 de Ecuador mediante la aplicación del software Gretl

Vol. 3, núm. 3 Esp., (2019)

Mariella Ginela Parrales Higuera; Verónica Alexandra Merchán Jácome; Washington Enrique Pazmiño Gavilanez; Lorena Isabel Muñoz Oviedo

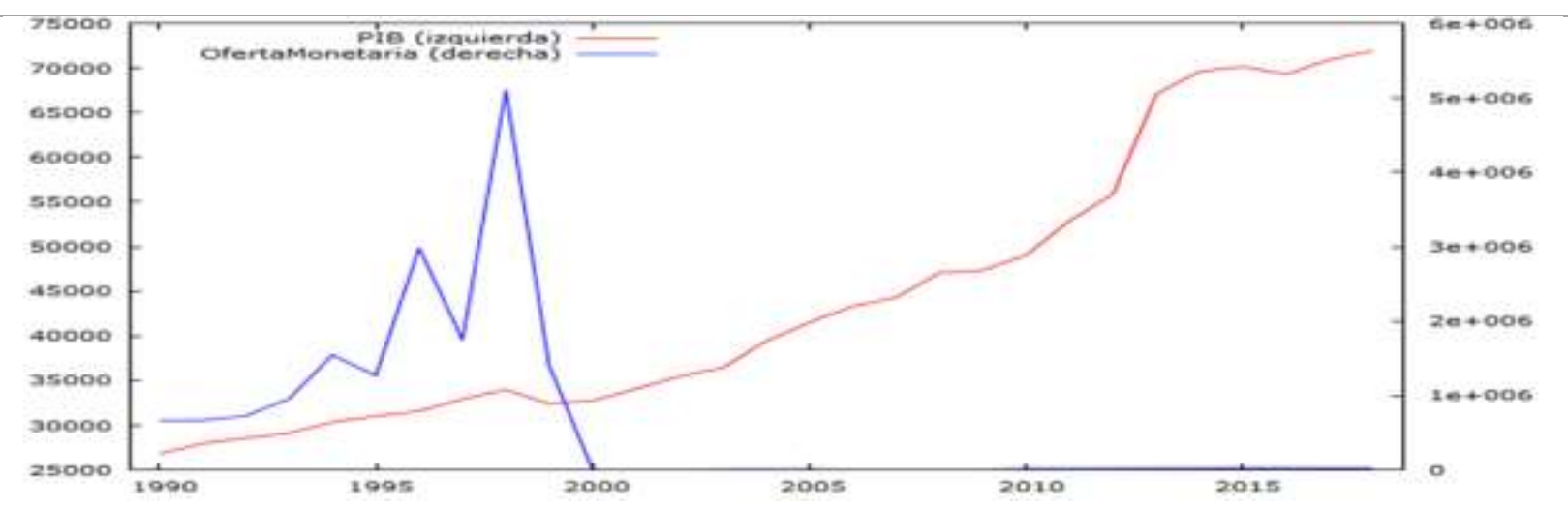

Fig. 13. PIB y Oferta Monetaria

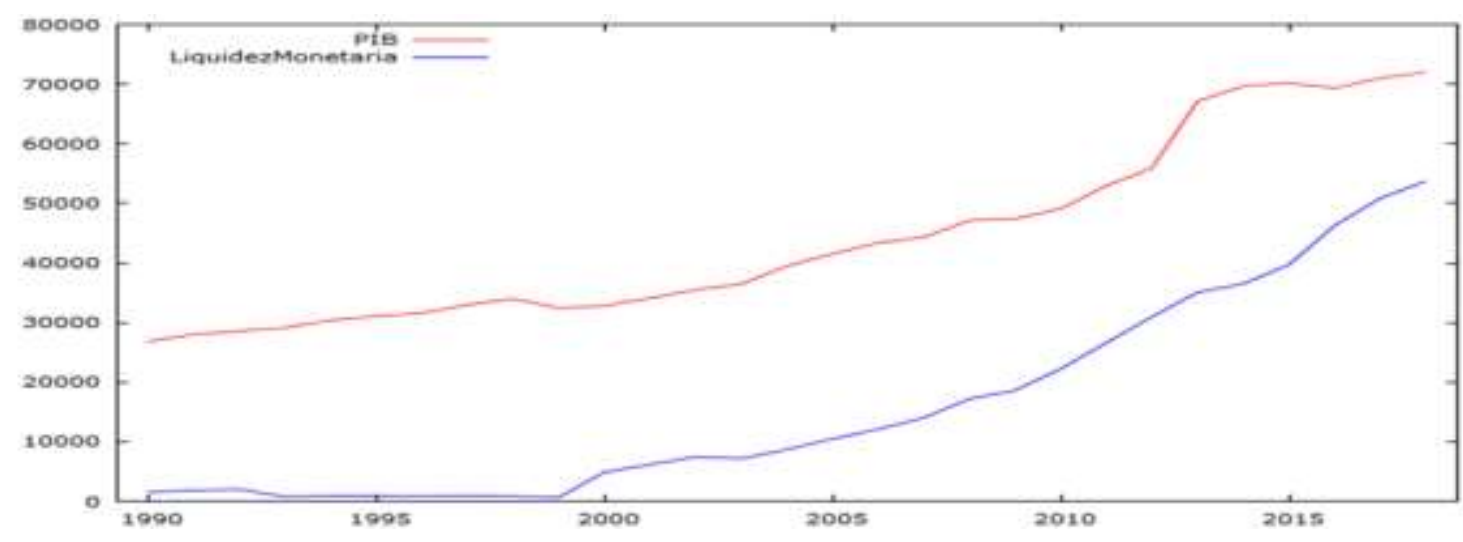

Fig. 14. Mínimos Cuadrados Ordinarios del modelo 
Modelo econométrico de la incidencia del Producto Interno Bruto con relación agregados monetarios, tasa de interés e inflación en el periodo 1990 2018 de Ecuador mediante la aplicación del software Gretl

Vol. 3, núm. 3 Esp., (2019) Mariella Ginela Parrales Higuera; Verónica Alexandra Merchán Jácome; Washington Enrique Pazmiño Gavilanez; Lorena Isabel Muñoz Oviedo

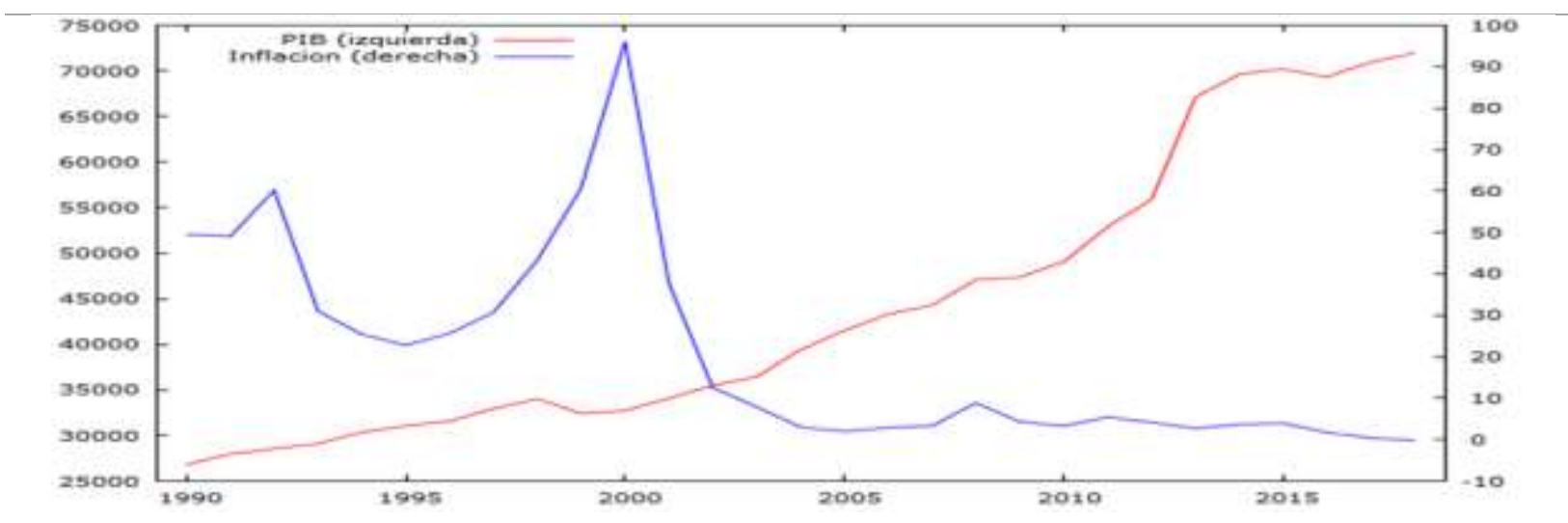

Fig. 15. PIB e Inflación

Como podemos observar en los cuatro gráficos la única cointegración que existe es mínima entre la variable dependiente PIB y la Liquidez Total. Por consiguiente, se trabajará con las dos variables con el fin de sacar la primera diferencia para convertir los datos en series de tiempo estacionarias, utilizando el método antes empleado de Dickey Fuller. 


\section{Modelo econométrico de la incidencia del Producto Interno Bruto con relación agregados monetarios, tasa de interés e inflación en el periodo 1990 -2018 de Ecuador mediante la aplicación del software Gretl}

Vol. 3, núm. 3 Esp., (2019)

Mariella Ginela Parrales Higuera; Verónica Alexandra Merchán Jácome; Washington Enrique Pazmiño Gavilanez; Lorena Isabel Muñoz Oviedo

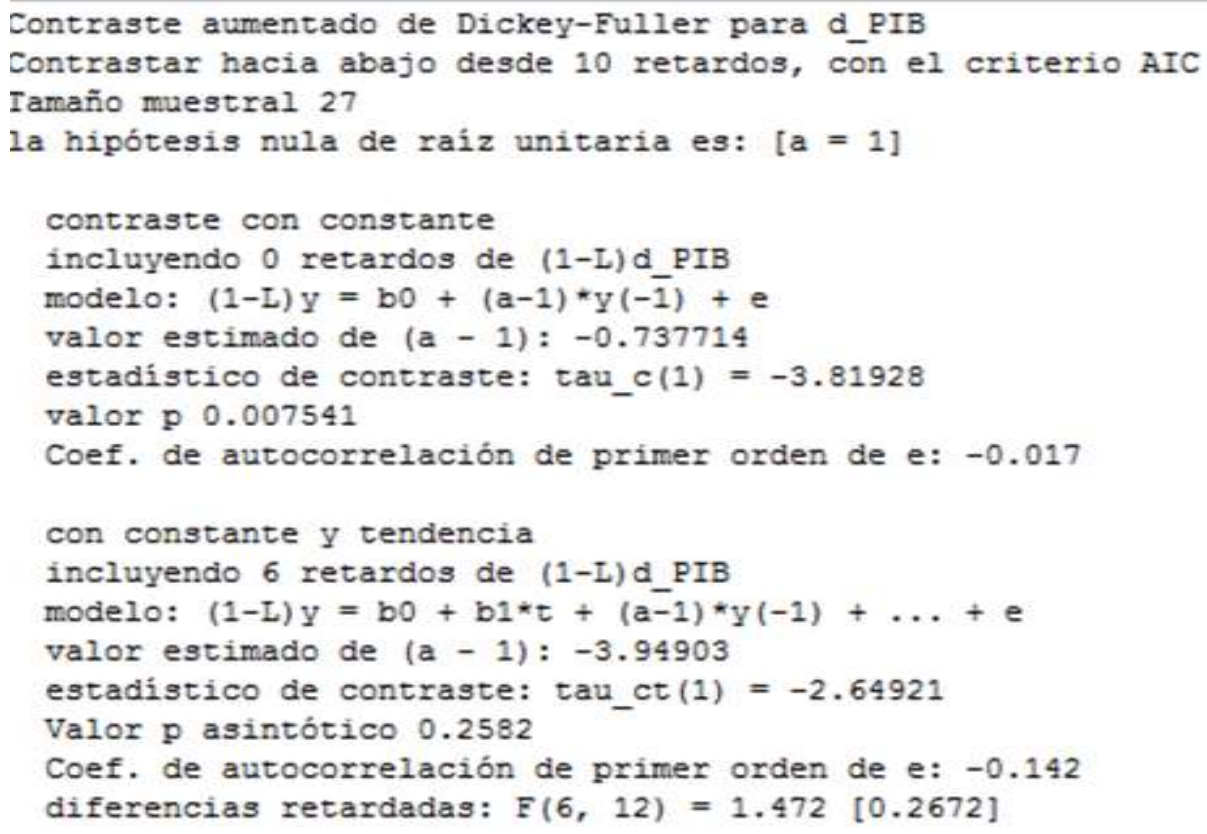

Fig. 16. Test de Dickey Fuller $1^{\circ}$ derivada PIB 


\section{Modelo econométrico de la incidencia del Producto Interno Bruto con relación agregados monetarios, tasa de interés e inflación en el periodo 1990 - 2018 de Ecuador mediante la aplicación del software Gretl}

Vol. 3, núm. 3 Esp., (2019)

Mariella Ginela Parrales Higuera; Verónica Alexandra Merchán Jácome; Washington Enrique Pazmiño Gavilanez; Lorena Isabel Muñoz Oviedo

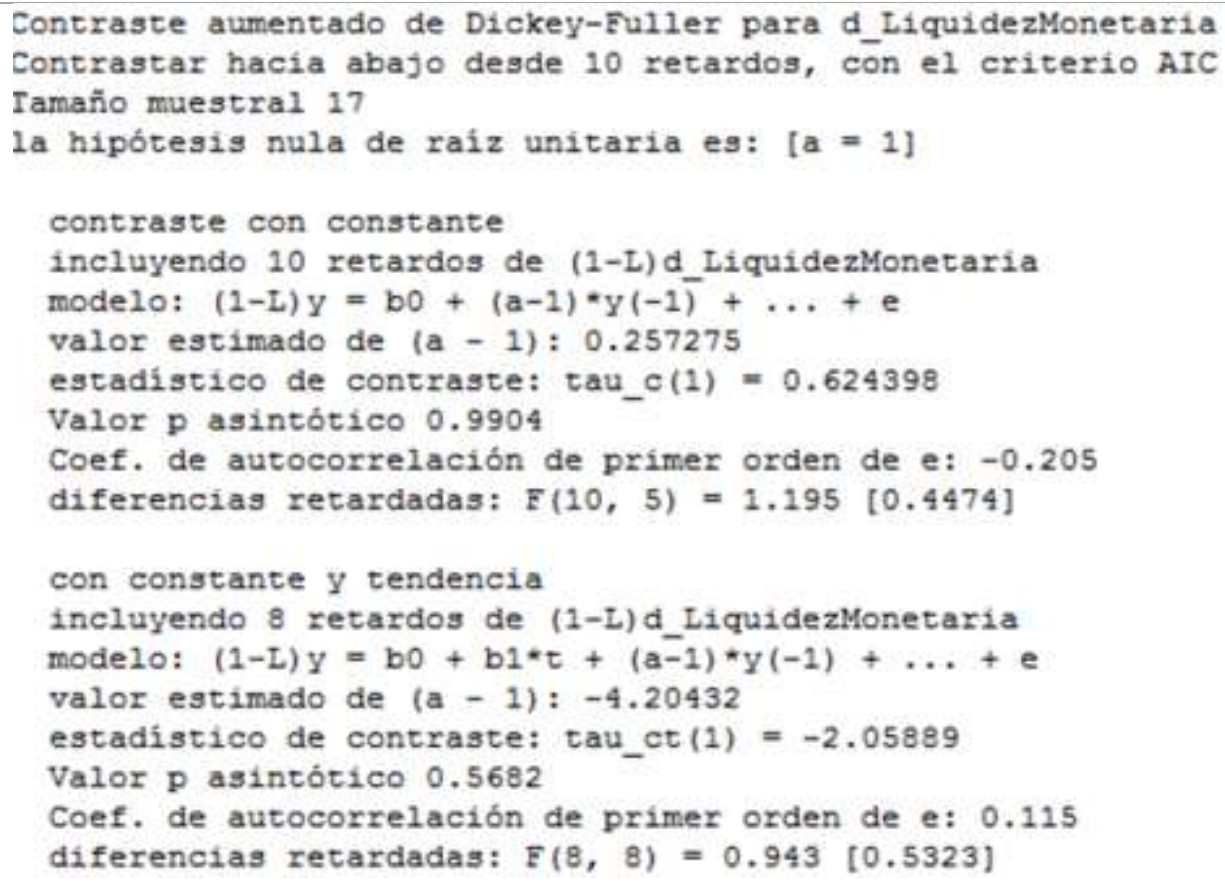

Fig. 17. Test de Dickey Fuller $1^{\circ}$ derivada Liquidez Total

Fuente: Gretl

Se puede observar en los dos gráficos anteriores que el estimador Rho es estadísticamente igual a 1, con un valor de probabilidad cercano a uno por lo que se rechaza la hipótesis nula. Por tanto, se tiene datos estacionarios.

Prueba de cointegración de johansen

Se puede apreciar que no existe estacionalidad en las dos variables sometidas a estudio, para lo cual se procede a realizar el Contraste de Engle-Granger. 


\section{Modelo econométrico de la incidencia del Producto Interno Bruto con relación agregados monetarios, tasa de interés e inflación en el periodo 1990 -2018 de Ecuador mediante la aplicación del software Gretl}

Vol. 3, núm. 3 Esp., (2019)

Mariella Ginela Parrales Higuera; Verónica Alexandra Merchán Jácome; Washington Enrique Pazmiño Gavilanez; Lorena Isabel Muñoz Oviedo

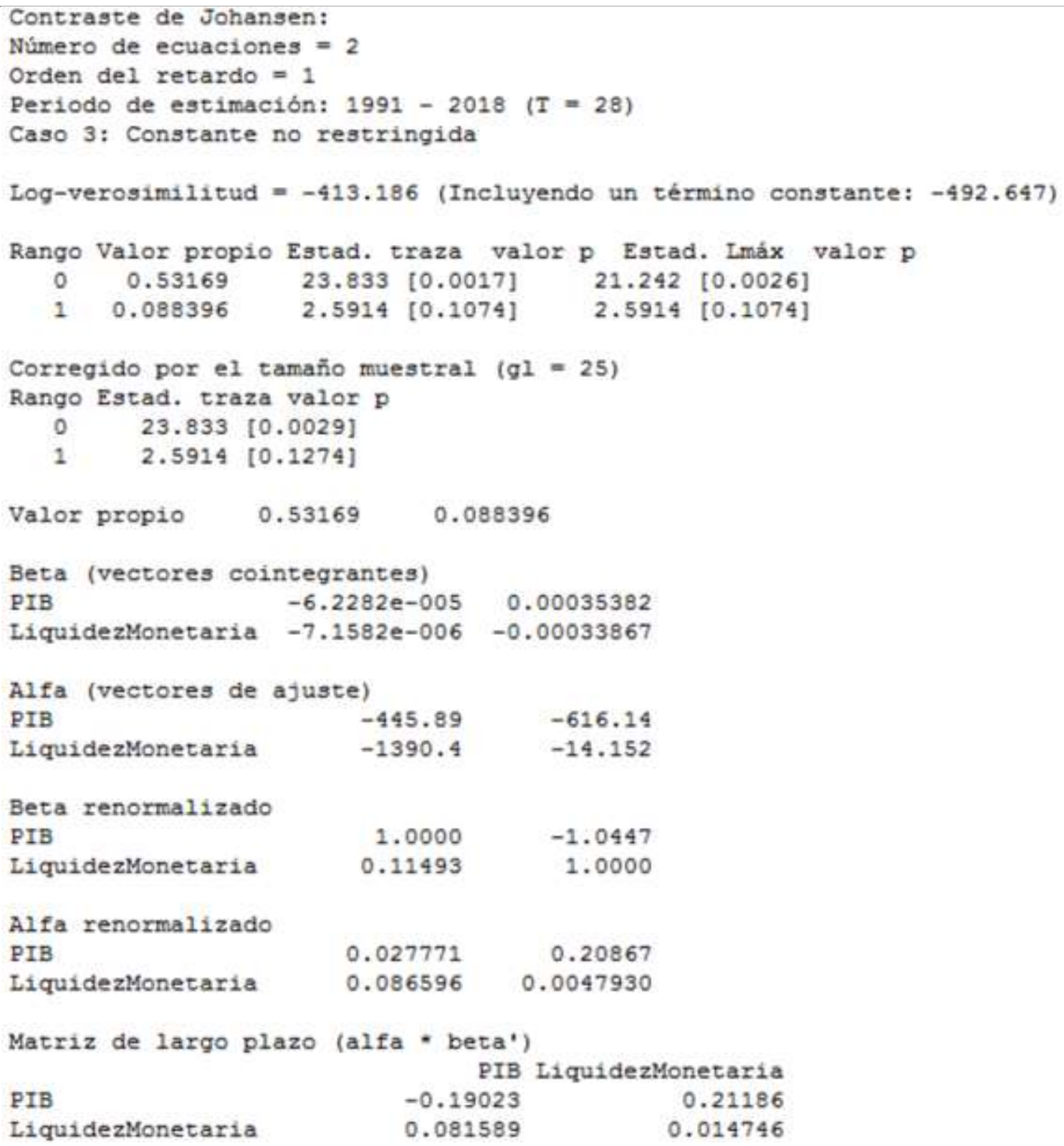

Fig. 18. Prueba de Cointegración de Johansen

Fuente: Gretl 


\section{Modelo econométrico de la incidencia del Producto Interno Bruto con relación agregados monetarios, tasa de interés e inflación en el periodo 1990 - 2018 de Ecuador mediante la aplicación del software Gretl}

Vol. 3, núm. 3 Esp., (2019)

Mariella Ginela Parrales Higuera; Verónica Alexandra Merchán Jácome; Washington Enrique Pazmiño Gavilanez; Lorena Isabel Muñoz Oviedo

Según las hipótesis se tiene:

$\mathrm{H} 0=$ No existen vectores de cointegración

H1 = Existe un vector de cointegración

Por tanto, la prueba de la traza se rechaza la hipótesis nula de no cointegración en favor de una relación de cointegración. En las matrices obtenidas, nos dirigimos inmediatamente a observar los valores presentes en la primera fila de beta re normalizada para lo cual después de realizar dicho contraste obtenemos una ecuación de: $\mathrm{Y}=1+1,0447 \mathrm{X} 1$ la cual es la ecuación normalizada.

\section{Discusión.}

La política monetaria es ciertamente un factor fundamental para explicar el comportamiento de la economía ecuatoriana ya que el modelo analizado muestra la relación del PIB con variables exógenas como son tasa de interés nominal, oferta monetaria, liquidez total e inflación las cuales permiten establecer el comportamiento que ha tenido económicamente nuestro país en los años de 1990 al 2018, además de tomar en cuenta los acontecimientos que han tenido un gran impacto en nuestra economía como fue la dolarización, la caída del precio del petróleo y las nuevas leyes establecidas por los distintos gobiernos, siendo que al correr el modelo en el software Gretl a través del uso del modelo ARIMA y los contrastes de Dickey Fuller y la prueba de Cointegración de Johansen se ha logrado evidenciar que la liquidez total es la variable que explica de mejor manera el PIB presentando proyecciones positivas con un crecimiento desde el año 2015 al 2021. 


\section{Modelo econométrico de la incidencia del Producto Interno Bruto con relación agregados monetarios, tasa de interés e inflación en el periodo 1990 -2018 de Ecuador mediante la aplicación del software Gretl}

Vol. 3, núm. 3 Esp., (2019)

Mariella Ginela Parrales Higuera; Verónica Alexandra Merchán Jácome; Washington Enrique Pazmiño Gavilanez; Lorena Isabel Muñoz Oviedo

La investigación abarcó el estudio de las políticas monetarias en base al PIB, Oferta Monetaria, Liquidez Total; conforme a los datos obtenidos del Banco Central del Ecuador se procedió a correr el modelo por ARIMA el cual una de las principales ventajas que encontramos de este método es que requiere de otras variables para explicar el objeto de estudio, como también los supuestos estadísticos, principalmente en cuanto a asignaciones de distribuciones de probabilidad como es en la serie de tiempo, por esta razón se usó el modelo antes mencionado para la elaboración del presente proyecto de investigación es por ello que todo está sustentado en la teoría aprendida y en la teoría de la política monetaria.

En Ecuador desde 1990 ha existido fluctuaciones en la economía ecuatoriana en el lapso los años en 1999 hubo el feriado bancario debido a esto en el 2000 Ecuador adopto el dólar americano y el Banco Central del Ecuador dejo de emitir la moneda el sucre ecuatoriano. Para el 2007 el nuevo gobernante el expresidente Econ. Rafael Correa realizado obras en el país como: educación, salud, vialidad e hidroeléctricas. Su objetivo fue dar una mejor calidad a los ciudadanos ecuatorianos.

\section{Bibliografía.}

Alonso, J. C. (2011). Tutorial Para Realizar La Prueba De Cointegración De Johansen Empleando Easyreg. Apuntes de Economía, (28), 28.

Banco Central del Ecuador. (2010). La Economía Ecuatoriana Luego de 10 Años de Dolarización. Direccion General de Estudios, 78. Retrieved from http://contenido.bce.fin.ec/documentos/PublicacionesNotas/Notas/Dolarizacion/Dolarizacio n10anios.pdf

García Camilo Pérez N, P. S. (2017). Desigualdad, inflación, ciclos y crisis en Chile *1 Inequality, inflation, cycles and crisis in Chile. Estudios de Economía, 44(2), 185-221. Retrieved from 


\section{Modelo econométrico de la incidencia del Producto Interno Bruto con relación agregados monetarios, tasa de interés e inflación en el periodo 1990 - 2018 de Ecuador mediante la aplicación del software Gretl}

Vol. 3, núm. 3 Esp., (2019)

Mariella Ginela Parrales Higuera; Verónica Alexandra Merchán Jácome; Washington Enrique Pazmiño Gavilanez; Lorena Isabel Muñoz Oviedo

https://scielo.conicyt.cl/pdf/ede/v44n2/0718-5286-ede-44-02-00185.pdf

Gujarati, D. N., \& Porter, D. C. (2013). Econometría. Mc Graw Hill (Vol. 53). https://doi.org/10.1017/CBO9781107415324.004

Herrera, G., \& Pérez Martínez, L. (2015). ¿Tiempos de crisis, tiempos de retorno? Trayectorias migratorias, laborales y sociales de migrantes retornados en Ecuador. Estudios Políticos (Medellin), (47), 221-241. https://doi.org/10.17533/udea.espo.n47a13

Mexiquense, E. C., Miguel, L., Horacio, G., La, L. O. S. E. D. E., Monetaria, P., El, E. N., ... Análisis, U. N. (2005). Los efectos de la política monetaria en el producto y los precios en México : un análisis econométrico, 65-101.

Montero, R. (2013). Variables no estacionarias y cointegración. Documentos de Trabajo En Economía Aplicada. Universidad de Granada, España, 1-8.

Renato, S. (2013). Gerenciamento de doenças utilizando séries temporais com o modelo ARIMA. Retrieved from http://www.scielo.br/scielo.php?script=sci_arttext\&pid=S167945082013000100024

Santana, G., \& Melo, J. (2016). Revista de Investigación y Desarrollo Minado de series de tiempo utilizando la metodología ARIMA Revista de Investigación y Desarrollo, 2(5), 21-31.

UNAM. (2013). MateMaticas financieras. Fca-Unam, 2, 1-41. Retrieved from http://ecampus.fca.unam.mx/ebook/imprimibles/informatica/matematicas_financieras/Unida d_2.pdf

Wooldridge, J. M. (2010). Introducción a la Econometría 4ta. Edición.

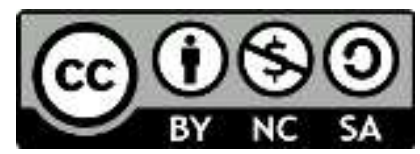

RECONOCIMIENTO-NOCOMERCIAL-COMPARTIRIGUAL

CC BY-NC-SA

ESTA LICENCIA PERMITE A OTROS ENTREMEZCLAR, AJUSTAR Y CONSTRUIR A PARTIR DE SU OBRA CON FINES NO COMERCIALES, SIEMPRE Y CUANDO LE RECONOZCAN LA AUTORÍA Y SUS NUEVAS CREACIONES ESTÉN BAJO UNA LICENCIA CON LOS MISMOS TÉRMINOS. 